\title{
Überlegungen W. Bangs zu komanisch *kär balık „Acipenser huso"
}

\author{
Michael Knüppel
}

Kassel

\begin{abstract}
The following article deals with W. Bang's attempts to give an etymology of an assumed Qoman *kar kär ballk "sturgeon" to which he traces back a name of the Sea of Azov given in the „Chiliads" by Tzétzis. The author of the present article explains *kar $\sim$ kär balık as a language taboo and gives an equivalent to this from Yakut (xatiïs ,sturgeon”).
\end{abstract}

Lösungen für in der Vergangenheit bereits längst aufgegebene etymologische Probleme finden sich nicht selten bei der Durchsicht älterer Aufzeichnungen, privater Notizen mitunter bereits lange verstorbener Vertreter der jeweiligen Disziplinen oder historischer Sprachmaterialien. Schon allein deshalb lohnt sich für den Etymologen der Blick in solche Unterlagen. Ein Beispiel mag hier eine kurze, belanglos erscheinende Notiz in einem Brief des Turkologen, Iranisten, Anglisten und Ural-Altaisten Johann Wilhelm 
(„Willi”) Max Julius Bang[-Kaup] (9.8.1869-8.10.1934) ${ }^{1}$ bieten. Dieser hatte - vor allem in seiner Zeit in Leuven/ Louvain - eine ausgedehnte Korrespondenz mit dem Iranisten Friedrich Carl Andreas (14.4.1846-3.10.1930) ${ }^{2}$ gepflegt, welche sich über mehrere Jahrzehnte hinzog. ${ }^{3}$ Gegenstand der erhaltenen Teile der späteren Korrespondenz (ab 1910) waren Bangs komanische Studien, an denen Andreas, da dieser in der Zeit seines Wirkens in Göttingen (ab 1903) dort als Iranist die „Orientalistik” (unter der man damals Studium und Lehre des Arabischen, Persischen und Osmanischen verstand) - und somit auch die noch junge Turkologie - vertrat, regen Anteil nahm.

In seinem Brief an Andreas (Brief vom 21.5.1911) schreibt Bang: „Gestern sandte ich Ihnen die Polowzer; ${ }^{4}$ sehen Sie sich bitte die Note zu p. 98 (čaquě) einmal vom Standpunkt des Irānisten genauer an. Ebenso empfehle ich Ihnen die Notiz zu ' $\alpha v \tau \alpha \kappa \alpha \iota \varsigma$, wobei ich nicht weiter kam, da mir Brehms Tierleben hier fehlt (: ist der Hausen mit „Enden”, „Nägeln” oder dgl. besetzt??).”

Diese Notiz ist einigermaßen irreführend, da sie sich auf mehrere, unterschiedliche Publikationen Bangs bezieht. Bei den „Polowzern” handelt es sich um seinen Beitrag „Zu der Moskauer Polowzischen Wörterliste," in der sich allerdings kein Hinweis auf den Hausen, Stör oder Sterlet resp. dessen mögliche Bennnung im Komanischen findet. „Die Notiz zu ' $\alpha v \tau \alpha \kappa \alpha$ lo $\varsigma$ ” wiederum findet sich in Anhang II zu seinem Aufsatz „Beiträge zur Kritik des Codex Cumanicus." "Etwas irritierend ist hier der Umstand, daß Bang entgegen seiner sonstigen Gewohnheit - Fragen hinsichtlich seiner bereits publizierten Schriften an Andreas richtet; d.h. er scheint nicht wirklich von einer befriedigenden Antwort ausgegangen zu sein und spielte allenfalls mit dem Gedanken mögliche weitergehende Feststellungen in geplanten künftigen Cumanica resp. in Anhängen zu

\footnotetext{
${ }^{1} \mathrm{Zu} \mathrm{W}$. Bang siehe - stellvertretend für die wahre Flut an Literatur zu seinem Leben und Werk - Schaeder (1929), de Vocht (1929), v. Gabain (1934), dies. (1974) u. Kononov (1974).

${ }^{2}$ Zu F. C. Andreas cf. v. Selle (1931), Littmann (1953), Kanus-Credé (1974), (1985) u. (1986), Röhrborn (2000).

${ }^{3}$ Die Reste des Briefwechsels zwischen W. Bang und F. C. Andreas finden sich in den Beständen der Handschriftenabteilung der Niedersächsischen Staats- und Universitätsbibliothek in Göttingen unter der Signatur „Cod. Ms. F. C. Andreas 1: 17” sowie im Archiv der Katholischen Universität Leuven.

${ }^{4}$ Bang (1911b).

5 Bang (1911a).
} 
selbigen zu bringen. Tatsächlich äußerte sich Andreas nahezu ein Jahr später (!) in einem Brief (vom 12.7.1912) zu dem Problem allerdings ohne etwas zur Lösung beitragen zu können: „Hier eine kleine Bemerkung zu der Moskauer Polowzischen Wörterliste S. 98 Anm. Das Wort für „Hammer” ist ursprünglich nicht türkisch, sondern aus dem Iranischen entlehnt, vgl. awestisch čakuš, neupers. čäkuš. Höchst wahrscheinlich ist auch das türkische Wort für „Löffel” iranisch, vgl. neupers. čämčä, wofür die Perser jetzt ausschliesslich das türkische qāšuq gebrauchen. Das $\underline{s}(\underline{u})$ vor dem $m$ der ersten Silbe in einzelnen türkischen Dialekten könnte auf ein vorauszusetzendes

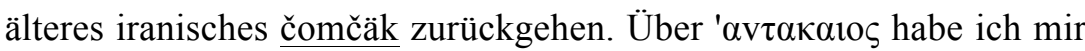
vergebens den Kopf zerbrochen. Seiner Lautgestalt nach kann das Wort sehr wohl iranisch sein, aber eine Erklärung will sich mir nicht darbieten."

In seinem Beitrag resp. dem II. Anhang zu seinem Aufsatz „Beiträge zur Kritik des Codex Cumanicus” äußerte sich Bang „ueber den Komanischen Namen der Maiotis" (dies = Azovsches Meer). Hierfür hat er einen Aufsatz von J. Marquart ${ }^{6}$ und die dort von diesem vorgenommene Interpretation einer Stelle aus den Chiliaden des Ioánnis Tzétzis zum Anlaß genommen. Bei dem von Tzétzis

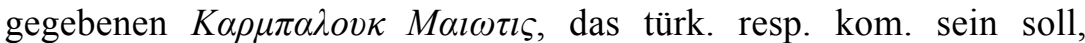
könnte, so Bang, von dem griech. Autor das kom. kermẽ $(=\text { kärmän })^{7}$

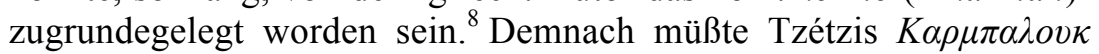
für seine Etymologie in $K \alpha \rho \mu+\pi \alpha \lambda o v \kappa$ aufgelöst haben. Bang wendet hier ein, daß dies nach „byzantinischem Schreibgebrauch” ein türk. $* k a r \sim k \ddot{a r}$ balık, an dessen Etymologie er sich versucht, wiedergibt ${ }^{9}$ und weist darauf hin, daß bei der Annahme eines Zusammenhanges mit kärmän in der ersten Silbe ein palataler Vokal anzusetzen ist. Was dann folgt, sind einige Bemerkungen zu dem aus dem Kom. nicht belegten kär: „In den Altaisch-Teleutischen Dialekten bedeutet kär ein Ungeheuer-grosses, Riesen-grosses Tier u.s.w. und Radloff's Wörterbuch führt kär palyq (II, p. 1083) in der Bedeutung "Wallfisch" auf” ${ }^{\prime 10}$ und der Deutung desselben als „Fischungeheuer”: „In dem

\footnotetext{
${ }^{6}$ Marquart (1910).

${ }^{7}$ Grønbech (1942), p. 140: kermen - „Stadt, "ciuitas"”'.

${ }^{8}$ Bang (1911a), p. 39.

${ }^{9}$ Ibd.

${ }^{10}$ Ibd., p. $39 \mathrm{f}$.
} 
Sagaischen Epos Altyn Pyrgan, Proben, II, ${ }^{11}$ p. 99; Vers 386, 387, 393; p. 103; Vers. 511, 518, 522 ist Kär Palyq eine Art mythologisches (?) Wesen. Die "echte" Sagaische Form Ker palyq findet sich ibid., p. 461, Vers 2809, p. 470, Vers 3107; zu ersterer Stelle die Anm. in der Uebersetzung, p. 469: "ein grosses Fischungeheuer." Wenn Herr Radloff das altaische kär palyq (Proben, I, p. 81 = Uebersetzung, pp. 84-85) durch "Wallfisch" übersetzt (vgl. auch W.B. a.a.O.), so ist dies nur ein Notbehelf, denn der Context beweist, dass es sich auch hier um ein fabelhaftes Fischungetüm handelt." ${ }^{\prime 2}$

Um seine Annahme eines Zusammenhangs zwischen dem Hausen und einem „Fischungeheuer” zu untermauern, gibt Bang dann noch geradezu wahnwizige Größenangaben für diesen Fisch: „Die um die Maiotis wohnenden Komanen können aber unter kär balyq nur den Hausen (acipenser huso), den pontisch-sarmatischen Fisch par excellence, verstanden haben, der in seinen grössten Exemplaren eine Länge bis zu 8 Metern erreicht." ${ }^{13}$ Derartige überzogene Größenangaben finden sich freilich auch in älteren Werken. Selbst in "Brehms Tierleben" werden unglaubliche Angaben geliefert. ${ }^{14}$ Tatsächlich erreicht der Europäische Hausen (Huso huso), der größte unter den Acipenseridae, eine maximale Länge von etwas über $5 \mathrm{~m}-$ was ja immerhin bemerkenswert genug, aber von Bangs Angaben noch immer erheblich entfernt ist.

Der Lösung des Problems der möglichen Benennung des Störs resp. Hausens im Kom. sehr viel näher als in seinem Beitrag war Bang mit seiner Notiz in dem Brief an Andreas (vom 21.5.1911). Er vermutete hier offenbar eine Bezeichnung für den Fisch, welche sich an dessen äußerer Erscheinung orientiert. Solche Benennungen kamen teils als fischereibedingte Tabuformen, ${ }^{15}$ teils als schlichte Bezeichnungen der Fische, Häufiger bei den Angehörigen solcher Gruppen, welche mit der Fischerei wenig zu tun hatten oder erst durch Kulturwandel bedingte Entwicklungen mit diesem in Berührung gekommen sind, vor. Bei turkophonen Gruppen zumeist in der Gestalt

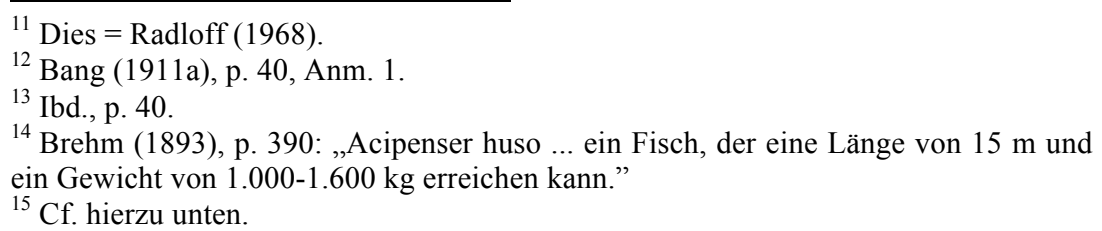


„X balık”, in der auf äußere Merkmale angespielt wird. So etwa im Neu-Uigurischen, dem, da im Siedlungsraum der Uiguren der Fischfang traditionell nicht $\mathrm{zu}$ den Erwerbszweigen der Einheimischen zählt, entsprechende Benennungen ebenso fehlen, wie Termini technici der Fischerei. Häufig sind die Bezeichnungen für Fische im Neu-Uig. aus dem Kirgisischen resp. von den kirgischen Bewohnern der Lop-Nor-Region übernommen, welche mit dem Fischfang vertrauter sind als ihre in der Vergangenheit bevorzugt Viehzucht treibenden Nachbarn. ${ }^{16}$ Bisweilen jedoch liegen auch einfach nur parallele Benennungen vor. Als Beispiel sei hier - um beim Beispiel zu bleiben - eine der Benennungen des Störs (oder genauer: des Acipenser baerii) aus dem Šorischen angeführt, wo dieser schlicht als sarty ballk (,gelber Fisch") bezeichnet wird. ${ }^{17}$ Dieselbe Benennung findet sich im auch Neu-Uig., allerdings für einen kleineren, karpfenartigen Fisch. ${ }^{18}$

In der Tat hätte sich für Bang ein Blick in „Brehm's Tierleben” gelohnt, da die hier in Frage kommenden Acipenseridae, wie alle Angehörigen ihrer Familie, tatsächlich Barteln und Kiemenreusendornen etc. aufweisen. ${ }^{19}$ Der Europäische Hausen etwa besitzt 17-36 solcher Dornen, d. h., hier könnte ohne weiteres ein „Nagel-Fisch” angenommen werden. Eine interessante Parallele dazu findet sich übrigens in C. H. Mercks „Sibirisch-Amerikanischem Tagebuch", welches sein Vf. als Teilnehmer der Billings-SaryčevExpedition in den Nordpazifik und die umliegenden Regionen (Kamčatka, Aleuten, Alaska etc.) in den Jahren 1788-1791 niederschrieb. Dort erwähnt Merck in einer Zusammenstellung der Fische des Viljuj-Flusses eine jakutische Benennung des Störs: „Seine

\footnotetext{
${ }^{16}$ Freundlicher Hinweis von Dr. Ablet Semet (Göttingen).

${ }^{17}$ Radloff IV (1911), col. 322. Bei Radloff findet sich über den Beleg aus dem Šor. hinaus eine ganze Reihe weiterer Beispiele für Benennungen für den Stör resp. Hausen, so bäkrä „Stör” (Čayataisch [Radloff IV, col. 1577]), bekrä „Sternstör, Sternhausen” (Kirgisisch [Radloff IV, col. 1577]), pilmai „Stör” (Sartischer Dialekt des Usbek. [Radloff IV, col. 1344]), šokur ,id.” (Kirgisisch [Radloff IV, col. 1024]), tugi $u$,id.” (Tobol-Dialekt [Radloff, III, col. 1432]) etc.

${ }^{18}$ Freundlicher Hinweis von Dr. Ablet Semet (Göttingen).

${ }^{19}$ Über die Schmelzschupper (Ganoidei), zu denen auch die Acipenseridae gezählt werden, lesen wir in Brehm (1893), p. 389: „Ihre Flossen sind oft, aber nicht immer, am Vorderrande mit einer einfachen oder doppelten Reihe von stachelartigen Tafeln oder Schindeln besetzt."
} 
Fische sind ... Sterlatten (echatyss), ... ${ }^{20}$ Hinter diesem verbirgt sich jak. xatï̈s „Stör” ${ }^{21}$ Es handelt sich dabei ganz unzweifelhaft um eine fischereibedingte Tabuisierung, ${ }^{22}$ da dies auf jak. xatiï „uneben, holprig; grannig, stachlig, dornig” zurückgeht. ${ }^{23} \mathrm{Zu}$ jak. xatï̈s „Stör” cf. auch dolgan. katïis ${ }^{24} \sim$ katïs „id.” ${ }^{25}$ Das ,überzählige” $e$ - das von Merck notiert wurde, dürfte entweder auf einer Verhörung beruhen oder auf einen russischsprachigen Informaten zurückgehen (cf. hierzu auch das bei Merck ebenfalls mit einem ,überzähligen” Anlautvokal gegebene jak. inuchely „Aland”26), da hier keiner der jak. Dialekte eine vokalisch anlautende Form zeigt. Zur jak. Benennung cf. auch einige der bei I. Hauenschild gegebenen Bezeichnungen: xatiïs/ xatiïs balik „Sibirischer Stör (Acipenser baeri), Sterlet (Acipenser ruthenus), Glattstör (Acipenser nudiventris)" und xatiïs toyon „Sibirischer Hausen (Huso dauricus). ${ }^{27}$

Die Form der Tabuisierung entspricht einem der im gesamten circumpolaren Eurasien gängigen Muster, d. h., die Bezeichnung eines Fisches oder Jagdtiers wird durch ein eigensprachliches Tabuwort, mit dem eigentlich eine Eigenschaft des Tieres beschrieben wird, ersetzt (so wird das [wilde] Rentier in den Jenissej-Sprachen als "das Langbeinige" bezeichnet, der Bär in tung. Idiomen als „,der Zottelige”, der Wolf als „der Gefräßige” etc.). Der Tabuisierung liegt die Vorstellung zugrunde, daß die Fische und Jagdtiere die Sprache der Menschen verstehen können und die Nennung derselben einen ausbleibenden Erfolg der Jäger und Fischer nach sich zieht.

Welche türk. Form Bang jedoch in seiner Anfrage an Andreas vorgeschwebt haben mag, bleibt sein Geheimnis. Er äußerte dort nur eine Vermutung in Gestalt einer Anfrage. Es könnten hier alle

\footnotetext{
${ }^{20}$ Dahlmann/ Friesen/ Ordubadi (2009), p. 92.

${ }^{21}$ Hierzu Knüppel (2011), Nr. 7.

${ }^{22}$ Es gilt hier das bei K. H. Menges auch zum Jagdtabu bei den Türken Sibiriens Ausgeführte (Menges [1932]).

${ }^{23}$ Cf. Stachowski (1993), p. 141. Der Erklärung bei Stachowski ist gegenüber der

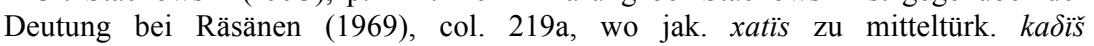
„Lederriemen”, Balkar., Kaz. Tel. etc. kajï̌s „Riemen” gestellt wird, eindeutig der Vorzug zu geben.

${ }^{24}$ Dem'janenko (1980), p. 124: 166; Stachowski (1993), p. 141.

${ }^{25}$ Bel'tjukova/ Koševerova (1987), p. 81; Stachowski (1993), p. 141.

${ }^{26}$ Dahlmann/ Friesen/ Ordubadi (2009), p. 117; cf. hierzu auch Knüppel (2011), Nr. 9; freundlicher Hinweis von Prof. Marek Stachowski (Kraków).

${ }^{27}$ Hauenschild (2008), p. 58.
} 
möglichen Wörter in Frage kommen. Zunächst natürlich das auch in Radloffs Wb. erscheinende, aus dem Ča (möglicherweise irrtümlich für kazık geschriebene [wie auch Radloff dort anmerkte]) karlk „Nagel, Pflock”. ${ }^{28}$ Vielleicht aber hat er auch an

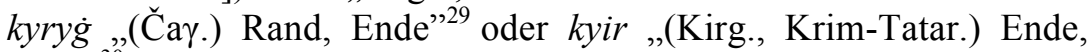
Ecke" ${ }^{\text {"30 }}$ gedacht - was natürlich allesamt nur Vermutungen sind. Es zeigt sich aber wie eingangs bemerkt, daß sich der Blick in Aufzeichnungen, wie die Briefe von Bang und Andreas in vielerlei Hinsicht lohnt und manchmal beiläufig geäußerte Vermutungen näher an der Lösung eines Problems sein können als das, was letztlich seinen Weg in die Publikationen gefunden hat.

\section{Literatur}

Bang, Willi. 1911a. Beiträge zur Kritik des Codex Cumanicus. In: Bulletin de l'Académie Royale de Belgique, Januar 1911, pp. $13-40$.

1911b. Zu der Moskauer Polowzischen Wörterliste. In: Bulletin de l'Académie Royale de Belgique, April 1911, pp. 91103.

Bel'tjukova, Natal'ja Petrovna/ Koševerova, Tamara Michajlovna. 1987. Materialy po dolganskomu jazyku voprosnika „Dialektologičeskogo atlasa tjurkskich jazykov Sibirii”. In: E. I. Ubrjatova et al. (Hrsgg.): Dialektnaja leksika tjurkskich jazykov Sibirii Novo sibirsk, p. 76-83.

Brehm, Alfred Edmund. 1893. Brehms Tierleben. Kleine Ausgabe für Volk und Schule. 2. Aufl. 3. Bd.: Kriechtiere, Lurche, Fische, Insekten, Niedere Tiere. Leipzig, Wien.

Dahlmann, Dittmar/ Friesen, Anna/ Ordubadi, Diana (Hrsgg.). 2009. Carl Heinrich Merck: Das sibirisch-amerikanische Tagebuch aus den Jahren 1788-1791. Göttingen.

\footnotetext{
${ }^{28}$ Radloff II (1899), col. 188

${ }^{29}$ Ibd., col. 740

${ }^{30}$ Ibd., col. 697.
} 
Dem'janenko, Z. P. 1980. Teksty (Ust'-Avam). In: Skazki narodov Sibirskogo Severa III. Tomsk, p. 85-146.

von Gabain, Annemarie. 1934. W. Bang Kaup 1869-1934. In: UJb 14, pp. 335-340.

. 1974. Persönliche Erinnerungen an W. Bang Kaup. In: Sprache, Geschichte und Kultur der altaischen Völker. Berlin, pp. 51-55.

Grønbech, Kaare. 1942. Komanisches Wörterbuch. Türkischer Wortindex zu Codex Cumanicus. Kopenhagen (Monumenta Linguarum Asiæ Maioris. Subsidia I).

Hauenschild, Ingeborg. 2008. Lexikon jakutischer Tierbezeichnungen.Wiesbaden (Turcologica 77).

Kanus-Credé, Helmart. 1974. Aus dem Nachlass von F. C. Andreas [I]. In: Iranistische Mitteilungen 8, pp. 42-75.

— 1985. Aus dem Nachlass von F. C. Andreas. II. Reisetagebuch Southampton - Port Said. In: Iranistische Mitteilungen 15, pp. 43-101.

1986. Aus dem Nachlass von F. C. Andreas. III. Briefe aus dem Iran. In: Iranistische Mitteilungen 16, pp. 1-83.

Knüppel, Michael. 2011. Jakutisches in C.H. Mercks „Sibirischamerikanischem Tagebuch" (1788/91). In: Turcica 43 (z. Zt. im Druck).

Kononov, Andrej Nikolaevič. 1974. W. Bang-Kaup. Zum hundertsten Geburtstag. In: Sprache, Geschichte und Kultur der altaischen Völker. Protokollband der XII. Tagung der PIAC 1969 in Berlin. Hrsg. v. Georg Hazai und Peter Zieme. Berlin, pp. 4749.

Littman, Enno. 1953. Andreas, Friedrich Carl. In: Neue Deutsche Biographie 1. Berlin, p. 284.

Marquart, Joseph. 1910. Karmpalouk, der „skytische” Name der Maiotis. In: Keleti Szemle XI, pp. 1-25.

Menges, Karl Heinrich. 1932. Jägerglaube- und gebräuche bei altajischen Türken. In: Le Muséon N.S. 45, pp. 85-90. 
Radloff, Wilhelm. 1868. Die Sprachen der türkischen Stämme SüdSibiriens und der dsungarischen Steppe. Abth. 1: Proben der Volkslitteratur der türkischen Stämme Süd-Sibiriens. Theil 2: Die Abakan-Dialecte (Der Sagaische, Koibalische, Katschinzische), der Kysyl-Dialect und der Tscholym-Dialect (Küarik). St. Petersburg.

.1893-1911. Versuch eines Wörterbuchs der Türk-Dialecte. 4 Bde. St. Petersburg.

Räsänen, Martti. 1969. Versuch eines etymologischen Wörterbuchs der Türksprachen. Helsinki (Lexica Societatis Fenno-Ugricae XVII: 1).

Röhrborn, Klaus. 2001. Friedrich Carl Andreas, 1846-1930. In: Göttinger Gelehrte. Die Akademie der Wissenschaften zu Göttingen in Bildnissen und Würdigungen 1751-2001. 1. Bd. Hrsg. v. Karl Arndt, Gerhard Gottschalk und Rudolf Smend. Göttingen, p. 312.

Schaeder, Hans Heinrich. 1929. Zu W. Bang's sechzigstem Geburtstag. In: UJb 9, pp. 181-187.

v. Selle, Götz. 1931. F.C. Andreas. In: Indogermanisches Jahrbuch 15 , pp. 366-367.

Stachowski, Marek. 1993. Dolganischer Wortschatz. Kraków (Zeszyty Naukowe Uniwersytetu Jagiellońskiego MLXXXVI. Prace Językoznawcze. Zeszyt 114).

de Vocht, Henri. 1929. Bibliographie der Arbeiten von Professor W. Bang Kaup. In: UJb 9, pp. 188-195. 
